\title{
Seasonal Adaptation of Vegetation Color in Satellite Images for Flight Simulations
}

\author{
Yuzhong SHEN, Jiang LI, Vamsi MANTENA, Srinivas JAKKULA
}

Department of Electrical and Computer Engineering, Old Dominion University, Norfolk, Virginia, USA.

Email: yshen@odu.edu, jli@odu.edu,vmant002@odu.edu, sjakk001@odu.edu

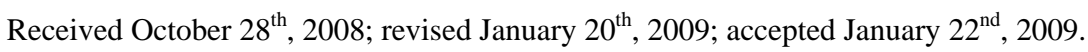

\begin{abstract}
Automatic vegetation identification plays an important role in many applications including remote sensing and high performance flight simulations. This paper proposes a novel method that identifies vegetative areas in satellite images and then alters vegetation color to simulate seasonal changes based on training image pairs. The proposed method first generates a vegetation map for pixels corresponding to vegetative areas, using ISODATA clustering and vegetation classification. The ISODATA algorithm determines the number of clusters automatically. We then apply morphological operations to the clustered images to smooth the boundaries between clusters and to fill holes inside clusters. Six features are then computed for each cluster and then go through a feature selection algorithm and three of them are determined to be effective for vegetation identification. Finally, we classify the resulting clusters as vegetation and non vegetation types based on the selected features using a multilayer perceptron (MLP) classifier. We tested our algorithm by using the 5-fold cross-validation method and achieved 96\% classification accuracy based on the three selected features. After the vegetation areas in the satellite images are identified, the proposed method then generates seasonal color adaptation of a target input image $Q$ based on a pair of training images $P$ and $P^{\prime}$, which depict the same area but were captured in different seasons, using image analogies technique. The final output image $Q^{\prime}$ has seasonal appearance that is similar to that of the training image $P^{\prime}$. The vegetation map ensures that only the colors of vegetative areas in the target image are altered and it also improves the performance of the original image analogies technique. The proposed method can be used in high performance flight simulations and other applications.
\end{abstract}

Keywords: pattern recognition, clustering, classification, satellite images, image analogies, texture synthesis, flight simulation

\section{Introduction}

Satellite images are routinely used as ground textures to simulate Earth surface and 3D features on Earth in terrain visualization, flight simulations, and many other applications. Texture mapping is a standard and powerful rendering technique used in modern computer graphics and it enhances realism and details with only a modest increase in computational complexity. However, it is common that satellite images of different regions were not captured in the same season and they exhibit substantial variations due to seasonal color changes of vegetation on the ground. Thus if satellite images captured in different seasons are used in flight simulations, the trainee would witness widely differing and constantly changing level of fidelity. In such systems, training effectiveness is compromised since the suspension of disbelief cannot be fully maintained. Therefore in many applications it is desired that satellite images captured in the same season be used and dynamically changed to a different season if needed.

This paper proposes a novel method that identifies vegetative areas in satellite images and then alters vegetation colors to simulate seasonal changes based on training image pairs. The proposed texture synthesis technique with the capability of automatic seasonal adaptation for vegetation consists of two steps: 1) identification of vegetation land cover from the satellite imagery; and 2) automatic texture synthesis for adaptation of the identified vegetation area to a different season. Vegetation area identification from satellite imagery includes image pixel clustering and cluster classification. We use the ISODATA algorithm [1] for the clustering task and utilize an MLP classifier to classify the clustered areas as vegetation and non-vegetation areas, based upon a set of selected features computed from the imagery. ISODATA 
is a divisive process which divides data into homogeneous clusters. There are two data passes in each iteration of the ISODATA algorithm. The first pass finds the means for each of the predefined number of clusters. The clusters are then split and merged in the second pass, depending upon their standard deviations, distance between means, cluster size and total number of clusters. Once the terminating criterion has been met, each data point is clustered into one of the predetermined clusters in terms of their distances to the cluster means. Usually, a post-processing step for smoothing cluster boundaries is needed due to noise in the image [2]. We apply morphological operations on the clustered images to smooth the boundaries between clusters and to fill holes inside clusters.

However, the ISODATA algorithm is an unsupervised learning process and land cover types of the resulting clusters are unknown. Therefore, the classification step is necessary. The next step is to identify the land cover type for each of the clusters. We compute a set of statistics for each of the clusters and train a classifier to classify the clusters into one of the land cover types. The statistics include mean and standard deviation of each spectral channel of the image. Note that not all the statistics are useful for the classification, we thus utilized a feature selection algorithm to identify the most effective features for the classification.

Training unnecessarily large classifiers can have side effects including the curse of dimensionality, convergence difficulties and large validation errors. In order to avoid these problems, feature selection is usually applied to generate a compact subset of features that leads to an accurate model for the given data. In feature selection, the algorithm often evaluates the fitness of candidate features first, it then searches for feature combinations with the goal of maximizing the fitness value. There are three popular different kinds of feature selection algorithms: filter, wrapper and embedded. A filter approach performs the fitness evaluation as a preprocessing step with no classifier involved such as FOCUS [3], Fisher criterion [4], weight-based [5] and RELIEF [6] algorithms. Filter approaches are usually very efficient but the selected feature subset may not perform well. A wrapper approach determines the fitness of a feature subset by actually training a classifier [7]. They usually have better performances at the cost of higher computational complexities. In the embedded approach, feature selection is done inside the algorithm itself. Examples include C4.5 [8] and CART [9]. In our previous work, we developed an efficient wrapper feature selection algorithm but it is as efficient as a filter algorithm [10]. We apply the feature selection algorithm to the six derived features and use a classification algorithm, also developed by the authors, to automatically identify land cover type of the clustered region $[11,12]$.
The proposed method then generates seasonal color adaptations of a target image $Q$ based on a pair of training images $P$ and $P^{\prime}$, using image analogies technique [13]. The training images $P$ and $P^{\prime}$ are images of the same area, but captured in different seasons. The final image $Q^{\prime}$ has seasonal appearance that is similar to that of the training image $P^{\prime}$. A similarity metric that is based on an approximation of the Markov random field is utilized [13]. The joint statistics of small neighborhoods within the training image and target image are used to measure the relationships between them. A Gaussian pyramid is constructed and several fast neighborhood searching algorithms are utilized. The vegetation map ensures that only colors of vegetative areas in $Q$ are altered.

The remainder of this paper is organized as follows. Section 2 describes the proposed method and its components, including ISODATA clustering, morphological operations, and feature selection and classification. Section 3 includes some experimental results and discussions. Finally conclusion is drawn in Section 4.

\section{Methods}

\subsection{ISODATA Clustering Algorithm}

Commonly used clustering algorithms in remote sensing include the K-means and ISODATA algorithms [1]. ISODATA stands for Iterative Self-Organizing Data Analysis, an advanced algorithm that can automatically adjust the number of clusters for a given data to be clustered. Both algorithms follow iterative procedures. In general, a clustering algorithm first assigns data points to arbitrary initial clusters. After that, the cluster means are recomputed and data points are reassigned. This process is repeated until the "change" between two consecutive iterations is sufficiently small. The "change" can be defined in several different ways, either by measuring the mean distances difference from one iteration to another or by the percentage of pixels that change cluster membership in consecutive iterations. The ISODATA algorithm is different from the K-means algorithm in that ISODATA dynamically adjusts cluster numbers while $\mathrm{K}$-means assumes that the number of clusters is fixed and is known a priori. The ISODATA algorithm adaptively adjusts the number of clusters by splitting and merging clusters. Clusters are merged if either the number of pixels in a cluster is less than a certain threshold or if the centers of two clusters are closer than a certain threshold. A cluster is split into two different clusters if the cluster's standard deviation exceeds a predefined value and the number of pixels is as twice as the threshold set for the minimum number of members.

The $R G B$ color space in which the original images are represented is converted into the $Y C_{b} C_{r}$ color space 
since the ISODATA clustering in the $Y_{b} C_{r}$ color space produces better results than in the $R G B$ color space. The components of the $Y C_{b} C_{r}$ color space represent luminance, blue chrominance, and red chrominance, respectively. The conversion from $R G B$ space to $Y C_{b} C_{r}$ space reduces cross-correlations between components. The ISODATA algorithm requires a set of pre-defined parameters to control its behavior [1]. These parameters were chosen experimentally in this paper. The following parameters were utilized.

$\begin{array}{cl}\text { K: } & \text { Number of desired clusters } \\ \text { I: } & \text { Maximum number of iterations allowed } \\ \text { P: } & \begin{array}{l}\text { Maximum number of pairs of clusters } \\ \text { that can be merged }\end{array} \\ \Theta_{N}: & \begin{array}{l}\text { Minimum number of samples in each } \\ \text { cluster (used for discarding clusters) }\end{array} \\ \Theta_{S}: & \begin{array}{l}\text { Maximum standard deviation for each } \\ \text { cluster (used for split operation) }\end{array} \\ \Theta_{C}: & \text { Minimum pairwise distances between } \\ & \text { cluster centers (used for merge operations) }\end{array}$

Among the 6 parameters listed above, the most important parameter used to control the algorithm is the number of samples in each cluster $\Theta_{N}$. The values of $\Theta_{N}$ vary from a few hundreds to a few thousands depending on the size of the image. Other parameters are mostly fixed. Typical values of $\mathrm{P}, \Theta_{C}, \Theta_{S}$ are 5, 0.001, and 10 , respectively. The maximum number of iterations $I$ has a fixed value of 20. Although the goal is to classify the image into vegetative and non-vegetative regions, we found it difficult for the ISODATA algorithm to generate two clusters corresponding to the vegetative and nonvegetative regions. The reason is that the trees and grass can have significantly different colors. Thus the number of desired clusters $K$ is set to 3. The above algorithm was successfully applied to satellite images to cluster images as different land cover types. The images shown in column (a) of Figures 18 and 18 are the original satellite images, while the images in column (b) are the clustering results of the ISODATA algorithm ${ }^{1}$, where three clusters are represented by white, black, and gray colors. However, the identities of these three clusters are not determined, that is, we don't know which cluster represents what ground feature.

\subsection{Morphological Operations}

The results of ISODATA are not perfect as the resulting clusters are not uniform and contain a lot of noise. The nonuniform clustering is because the variations in the original input images and the imperfectness of the algo- rithms (ISODATA) used. The initial clustering result can be improved by morphological operations.

Closing and opening [14] are commonly used morphological operations in image processing to remove or expand gaps in an image. In this paper, closing operation is performed with size of the structuring element equal to the maximum size of the hole or gap in one cluster. There are other regions in the image where there may exist small gaps between vegetation regions. To retain these small gaps, opening operation is performed on the image obtained in the previous stage with the size of structuring element equal to the maximum size of the gap or hole. By using these operations the clustered image also gets smoothed. The sequence of operations and size of structuring elements may vary depending upon the types of the clustered images. The images in column (c) of Figures 1 and 2 show the results of morphological processing.

\subsection{Feature Selection and Classification}

A cluster in a clustered image is a group of pixels having similar characteristics. However, vegetation type of a cluster is unknown, i.e., we do not know if a cluster is grass or sand. In this paper, we use supervised learning to recognize the identity of the clusters. We classify the clusters into two types: vegetation and others. We compute six features for each of the clusters including mean and standard deviation of $Y, C_{b}$ and $C_{r}$ channels. Therefore, we have six features for each cluster.

These six features are derived heuristically and they are not equally important. We utilize a feature selection algorithm developed in our previous work [10] to select a compact set of features that leads to an accurate model based on the available data. The feature selection algorithm 1) determines an appropriate piecewise linear classifier (PLC) model for the given data set, 2) applies the orthonormal least squares procedure to the PLC model and searches for useful feature subsets using a floating search algorithm. The floating search prevents the nesting effect. The feature selection algorithm is computationally efficient because only one data pass is required. The feature selection algorithm is explained in detail as follows.

\section{Step 1: Design a piecewise linear classifier for given data}

Piecewise linear classifier approximates the general Bayes discriminant. The available data is divided into a set of clusters where a local linear model is obtained for each cluster, by solving a set of linear equations. Neural classifiers including the PLC are usually designed by minimizing the standard training error,

$$
E=\sum_{i=1}^{N_{c}} E(i)=\frac{1}{N_{v}} \sum_{i=1}^{N_{c}} \sum_{p=1}^{N_{v}}\left[t_{p}(i)-y_{p}(i)\right]^{2},
$$


where $N_{c}$ is the number of classes and $E(i)$, the mean-squared error for the ith output. Here $t_{p}(i)$ denotes the $i$ th desired output for the $p$ th input pattern, $y_{p}(i)$ denotes the $i$ th observed output for the $p$ th input pattern, and $N_{v}$ denotes the total number of data patterns. In the PLC, $y_{p}(i)$ is the output from the piecewise linear network,

$$
y_{p}(i)=\sum_{j=1}^{N+1} w^{(q)}(i, j) x_{p}^{(q)}(j),
$$

where $N$ is the number of features, $w^{(q)}(i, j)$ denotes the model weight to the ith output from the $j$ th feature in the $q$ th cluster, $x_{p}^{(q)}(N+1)$ is the $j$ th feature in the $q$ th cluster, and $x_{p}^{(q)}(N+1)$ is the bias term which equals one. We assume that $t_{p}\left(i_{c}\right)=b$ and $t_{p}\left(i_{d}\right)=-b$ where $i_{c}$ denotes the correct class number and $i_{d}$ any incorrect class number for the current data pattern. If $i_{c}=\operatorname{argmax}_{i} y_{p}(i)$, we say the PLC classifies the current pattern correctly. Otherwise, a classification error is counted.

Step 2: Search a list of good feature combinations using the floating search algorithm

We design a floating search method through Piecewise Linear Orthonormal Least Square (PLOLS) procedure in this section. The PLOLS procedure utilizes the modified Schmidt procedure to make each features in each cluster orthonormal. This procedure passes through the data set once, and all information needed for searching good combination of features is stored in the auto-correlation and cross-correlation matrices. Therefore, our feature selection algorithm is very efficient.

Based on Equations (1) and (2), the modified desired output may be represented in a matrix form as

$$
\mathbf{t}^{\prime}=\mathbf{x}^{(q)} \mathbf{w}^{(q)}+\Xi^{(q)},
$$

where each row in matrix $\mathbf{X}^{(q)}$ represent one feature vector that was assigned to the $q$ th cluster, $\mathbf{W}^{(q)}$ denotes weight matrix in the $q$ th cluster, and $\Xi^{(q)}$ are residual errors in the qth cluster. We apply the modified Schmidt procedure to each cluster, yielding the piecewise linear orthogonal (PLO) system [10]

$$
\mathbf{t}^{\prime}=\Theta^{(q)} \mathbf{A}^{(q)} \mathbf{w}^{(q)}+\Xi^{(q)}=\Theta^{(q)} \mathbf{w}_{o}^{(q)}+\Xi^{(q)} .
$$

Once the model (4) is available, fitness values for features are easy to compute using the orthonormal weights $\Theta^{(q)}$, which are calculated from the auto-and cross-correlation matrices through the modified Schmidt procedure. Note that the original data set is not touched

\footnotetext{
${ }^{1}$ We utilized a fast implementation of the ISODATA algorithm by professor David Mount from the University of Maryland. See online code: http://www.cs.umd.edu/ mount/Projects/ISODATA/.
}

after the matrices accumulated, leading to a very efficient feature selection algorithm. See [10] for more details.

\section{Algorithm Description}

Suppose we select $N_{s}$ features from $N$ available ones, the overall feature selection algorithm can be described as follows,

1) Using the trial and error method to determine an appropriate number of clusters, $N_{c}$, which will be used in the PLC.

2) Design an $N_{c}$ cluster PLC for the data by solving a set of linear equations for each cluster.

3) Search a list of good feature combinations using the PLOLS procedure.

Advantages of the proposed algorithm are as follows: 1) It selects features rather than a combination of all the features such as those selected by transformation based methods (PCA, Wavelet), 2) It considers interactions among features and measures the correlations via the amount of explained variance by features, 3) It is computationally efficient, and 4) The algorithm produces a list of best combinations that contain different numbers of features, users then have the flexibility to choose one based on performance.

\section{Step 3: Classification}

After the compact set of features are selected, we use an MLP classifier to classify the cluster to one of the two classes [11,12]. The classifier utilized a new objective function that had more free parameters than the classical objective functions, and used an iterative minimization technique to solve multiple sets of numerically ill-conditioned linear equations. An enhanced feedforward network training algorithm was also used to reduce a separate error function with respect to hidden layer weights. The MLP classifier is explained in detail as follows.

We are given a set of $N_{v}$ training patterns $\left(x_{p}, t_{p}\right)$ where the $p$ th input vector $x_{p}$ and $p$ th desired output vector $t_{p}$ have dimension $N$ and $N_{c}$ respectively. A three layer, fully connected MLP networks with sigmoid activation function for the hidden layer is used. For the $p$ th pattern, the $j$ th hidden unit net and activation functions are

$$
\begin{gathered}
\operatorname{net}_{p}(j)=\sum_{k=1}^{N+1} w(j, k) \cdot x_{p}(k), \\
O_{p}(j)=f\left(\operatorname{net}_{p}(j)\right)=\frac{1}{1+\exp ^{\left(-\operatorname{net}_{p}(j)\right)} .}
\end{gathered}
$$

The ith observed output is

$$
y_{p}(i)=\sum_{k=1}^{N+1} w_{o i}(i, k) \cdot x_{p}(k)+\sum_{j=1}^{N_{h}} w_{o h}(i, j) \cdot O_{p}(j)
$$

where $w_{o i}(i, k)$ and $w_{o h}(i, j)$ are weights connecting to the $i$ th output unit from the $k$ th input and $j$ th hidden unit re- 
spectively. For the $j$ th hidden unit and $p$ th pattern, the desired net function net $_{p d}(j)$ is constructed as

$$
n e t_{p d}(j) \cong n e t_{p}(j)+Z \cdot \delta_{p}(j),
$$

$Z$ is the learning rate and $\delta_{p}(j)$ is the delta function of the $j$ th hidden unit and is defined as

$$
\delta_{p}(j)=f^{\prime}\left(\operatorname{net}_{p}(j)\right) \sum_{i=1}^{N_{c}} \delta_{p o}(i) w_{o}(i, j),
$$

where $\delta_{p o}(i)$ is the delta function of the $i$ th output layer,

$$
\delta_{p o}(i)=t_{p}(i)-y_{p}(i)
$$

The hidden weights are updated as

$$
w(j, k) \leftarrow w(j, k)+Z \cdot e(j, k),
$$

where $e(j, k)$ is the hidden weight change. With the basic operations and (5-11), we can use the following equation to solve for the changes in the hidden weights,

$$
n e t_{p d}(j)+Z \cdot \delta_{p}(j) \cong \sum_{k=1}^{N+1}[w(j, k)+Z \cdot e(j, k)] \cdot x_{p}(k) .
$$

We obtain

$$
\delta_{p}(j) \cong \sum_{k=1}^{N+1} e(j, k) \cdot x_{p}(k) .
$$

Before solving (13) in the least squares sense, an objective function for the $j$ th hidden unit is defined as

$$
E_{\delta}(j)=\sum_{p=1}^{N_{v}}\left[\delta_{p}(j)-\sum_{k=1}^{N+1} e(j, k) x_{p}(k)\right]^{2} f^{\prime}\left(\text { net }_{p}(j)\right),
$$

which is minimized with respect to $e(j, i)$ using the conjugate gradient method and we obtain the hidden weights change $e(j, k)$, we then update the hidden weights by performing (11). Refer to our previous publications $[10,11,12]$ for more details of the feature selection and the MLP classifier training algorithms.

As suggested by reviewers, we also apply the support vector machine and the nearest neighbor classifiers on the selected features. Classification results from different classifiers will be compared.

\subsection{Seasonal Color Adaptation}

After the vegetation map is obtained, the colors of the vegetation area can be adapted to other seasons utilizing the image analogies technique [13]. Image analogies generates an output image $Q^{\prime}$ that is related to an input image $Q$, imitating the relationship between a pair of training images $P^{\prime}$ and $P$. The image analogies process can be represented mathematically as:

$$
P: P^{\prime}:: Q: Q^{\prime},
$$

where the symbol :: denotes that the relationship between $P$ and $P^{\prime}$ is similar to that between $Q$ and $Q^{\prime}$. $P^{\prime}$ and $Q^{\prime}$ can be thought of as filtered versions of $P$ and $Q$, respectively. For the seasonal color adaptation in our work, the training pair $P$ and $P^{\prime}$ are images of the same area but captured in different seasons, the target image $Q$ is captured in the same season as is $P$, then the output image $Q^{\prime}$ would look as if it is captured in the same season as is $P^{\prime}$.

The image analogies technique assumes that the two training images are registered; that is, the colors at and around any given pixel $p$ in $P$ correspond to the colors at and around the same pixel $p$ in $P^{\prime}$. A similarity metric based on an approximation of a Markov random field is utilized by the image analogies technique [13]. Specifically, the joint statistics of small neighborhoods within the training image and target image are used to measure the relationships between them. Figure 3 shows an example of the training image pair $P$ and $P^{\prime}$, the target input image $Q$, and the output image $Q^{\prime}$. For each pixel $q$ in the image $Q$, the image analogies technique searches for a corresponding pixel $p$ in the image $P$ such that the small neighborhoods of $p$ and $q$ have similar statistics. The two orange squares surrounded by $5 \times 5$ windows in Figures 3(a) and (c) illustrate such matching pixels in $P$ and $Q$, respectively. The pixel $p$ in $P^{\prime}$ is then copied to pixel $q$ in $Q^{\prime}$, since the images $P$ and $P^{\prime}$ are registered.

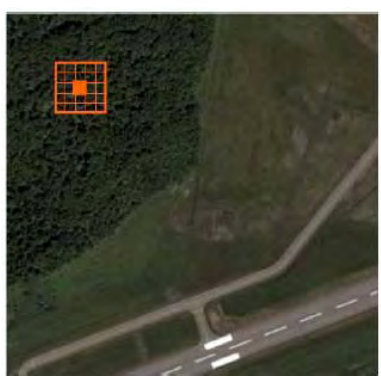

(a) $P$

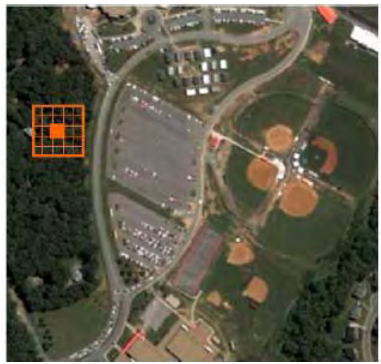

(c) $Q$

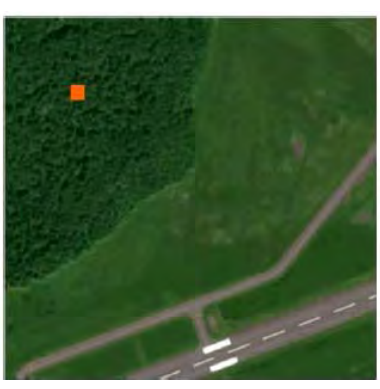

(b) $P^{\prime}$

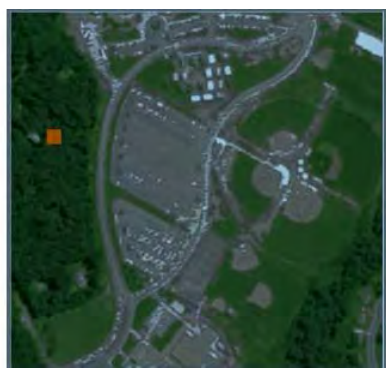

(d) $Q^{\prime}$
Figure 3. Image analogies. (a) and (b) are the training image pair $P$ and $P^{\prime}$. (c) is the target image $Q$. For each pixel $q$ in the target image $Q$, a matching pixel $p$ in $P$ is found such that the neighborhoods of $p$ and $q$ ( $5 \times 5$ windows in (a) and (c)) have similar statistics; then the pixel that has the same location as $p$ in $P^{\prime}$ is copied to the pixel in $Q^{\prime}$ in (d), which has the same location as $q$. The pixels in $Q^{\prime}$ are generated in a scan-line order. 
That is, the pixel at the position labeled by the orange square in Figure 3(b) is copied to the pixel at the position labeled by the orange square in Figure 3(d). The pixels in $Q$ are processed in a scan-line order; thus the pixels in $Q^{\prime}$ are generated in the same order.

The joint statistics of a small neighborhood centered at a pixel are concatenation of the feature vectors of all the pixels in the neighborhood. The feature vector of each pixel can consist of $R G B$ color components, luminance, various filter responses, etc. In this work, only $R G B$ color components are included in the feature vector. The efficient implementation of image analogies technique makes uses of multi-scale representation and fast neighborhood search methods [13]. The multi-scale representation first constructs Gaussian pyramids of images $P, P^{\prime}$, and $Q$ with $I$ levels; the synthesis then proceeds from coarsest to finest resolution, computing a multi-scale representation of $Q^{\prime}$, one level at a time. The pseudocode of the algorithm [13] is shown below.

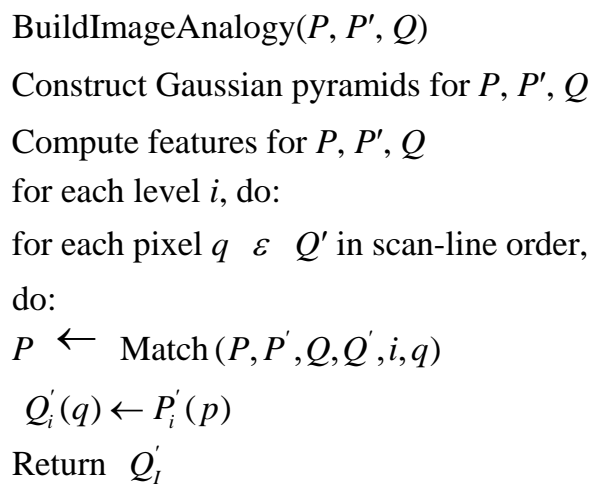

$Q^{\prime}{ }_{I}$ has the finest resolution in the Gaussian pyramid and it is the final output. The Match() function in the pseudocode uses fast neighborhood search algorithms, including the approximate-nearest-neighbor-search [15, 16] and coherence search [17].

As noted in their original work [13], although image analogies are an effective image synthesis method, it is not perfect. This can be clearly seen in the example output image $Q^{\prime}$ shown in Figure 3(d). The original target image $Q$ (Figure 3(c)) has some ground objects with brown colors, but these objects' colors are changed to gray in $Q^{\prime}$. This is an undesired effect since these ground objects don't change their colors in different seasons. To restrict color changes to vegetative areas only, the vegetation map generated in the previous section is utilized. The vegetation map of $Q$ is shown in Figure 4(a). Utilizing this vegetation map, non-vegetative regions in $Q$ are copied back to $Q^{\prime}$ to form the final image shown in Figure 4(b). Our current implementation utilized the code from [18]. Our future implementation will utilize the vegetation map in the neighborhood search process, i.e., the search is restricted to the vegetative areas of $P$ and $Q$ only. This will certainly improve the output image quality since the probability of erroneous neighborhood match is greatly reduced. The computational time will also be significantly reduced.

\section{Results and Discussion}

The results of ISODATA clustering, morphological operations, and classification are shown in columns (b), (c), and (d) in Figures 1 and 2. To study the effectiveness of the ISODATA algorithm, we have applied it to the 120 satellite images. A total of 120 satellite images have been utilized in this study. We computed the six features for each of the clusters and assigned one class type (vegetation or non-vegetation) to each of the clusters. The feature selection algorithm selected three of the features (means of the $Y$ and $C_{b}$ channels and standard deviation of the $C_{b}$ Channel) and the other three features turned out to be not useful. To test if a classifier can automatically identify vegetation type, we used the 5-fold cross-validation method on the 120 images using three different classifiers: the MLP classifier described previously, the support vector machine (SVM) and the k-nearest neighbor $(k-N N)$ classifier. The MLP has 3 inputs, 1 hidden unit and 2 outputs, and the classifier was trained by the method described in Subsection 2.3.

Classification results are listed in Table 1. It is clear that the MLP classifier achieved the best classification accuracy, 96\%, which is slightly better than that of the 1NN classifier. The SVM classifier is the worst in terms of

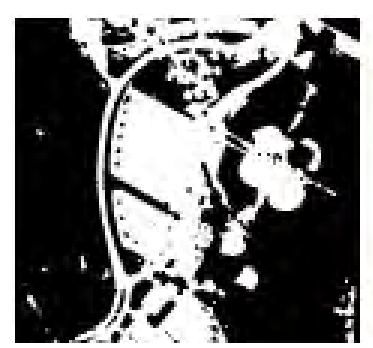

(a)

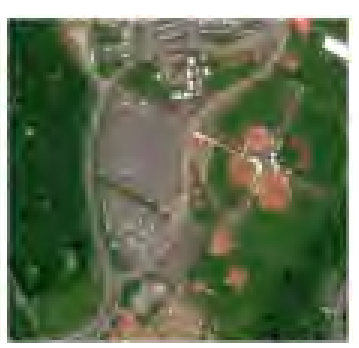

(b)
Figure 4. (a) Vegetation map of Figure 3(c). (b) Final seasonal color adaptation of Figure 3(c) based on the training pair in Figure 3(a) and (b).

Table 1. Classification results by three different classifiers.

\begin{tabular}{cccccc}
\hline Algorithm & MLP & SVM & 1-NN & 3-NN & 5-NN \\
\hline Accuracy\% & 96 & 91.6 & 94.5 & 93.6 & 92.5 \\
\hline
\end{tabular}




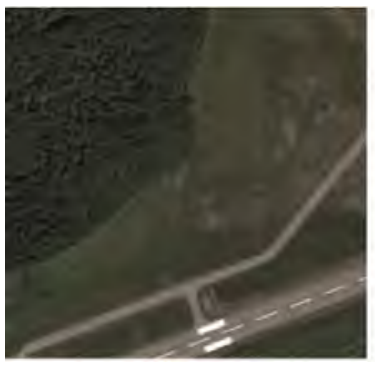

(a)

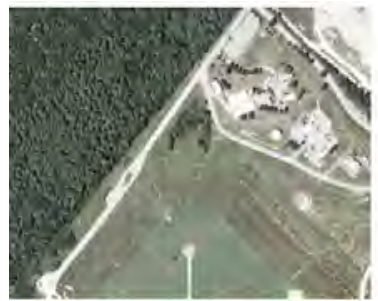

(a)

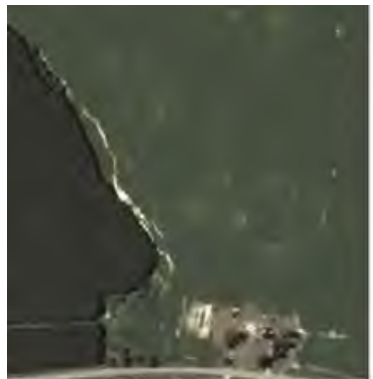

(a)

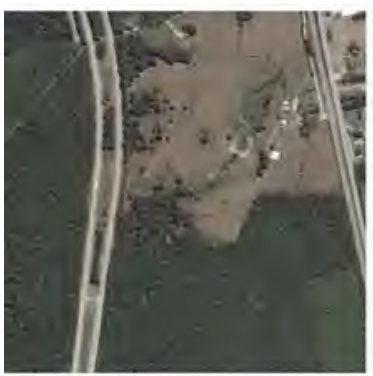

(a)

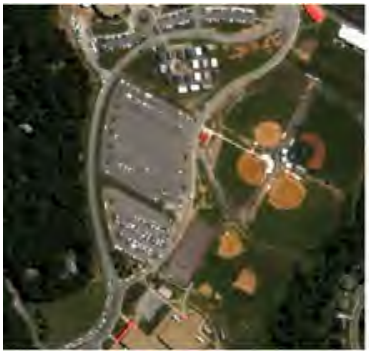

(a)

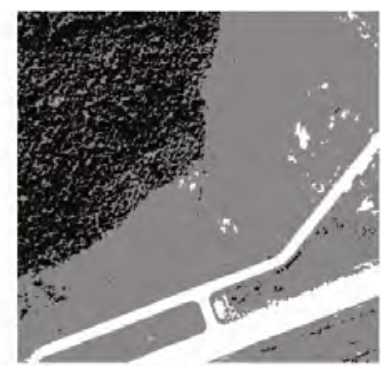

(b)

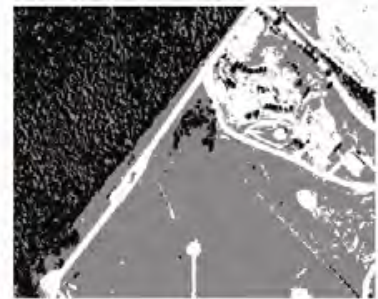

(b)

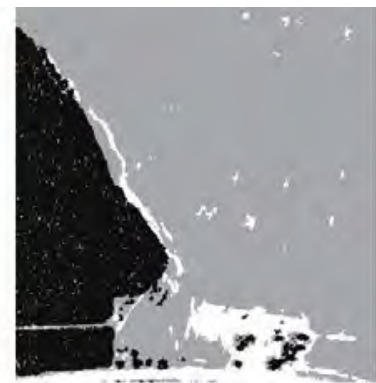

(b)

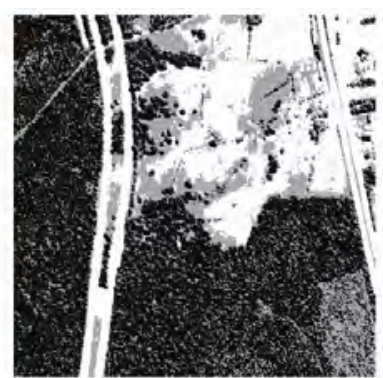

(b)

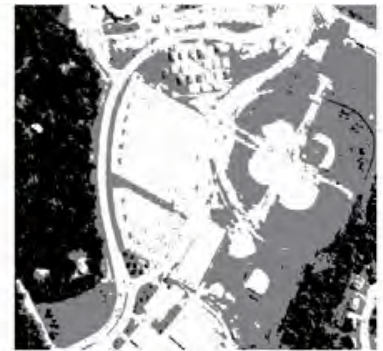

(b)

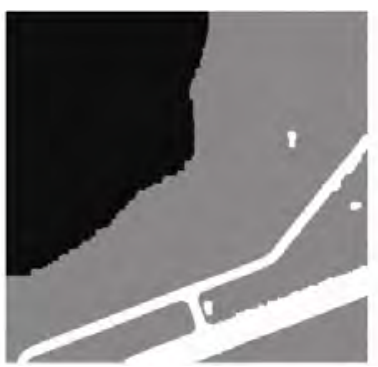

(c)

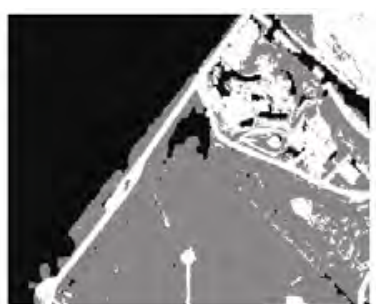

(c)

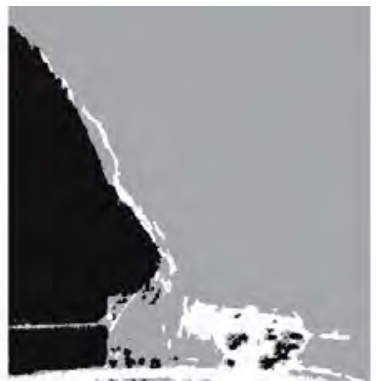

(c)

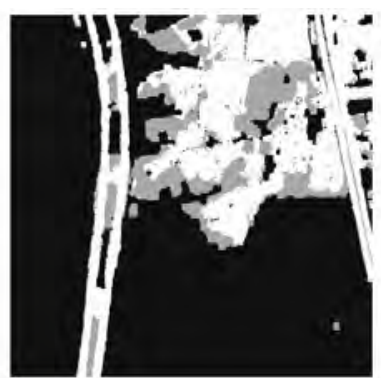

(c)

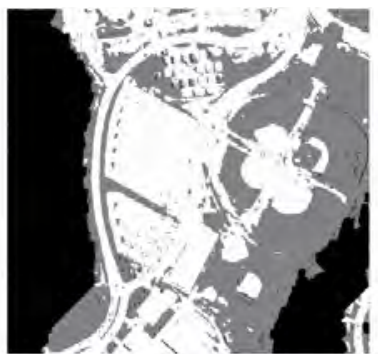

(c)

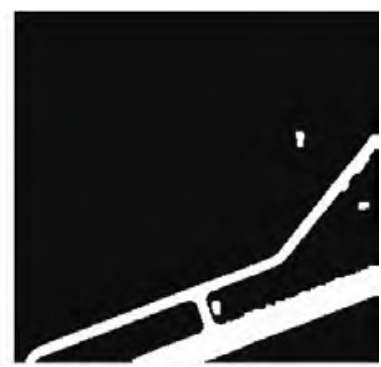

(d)

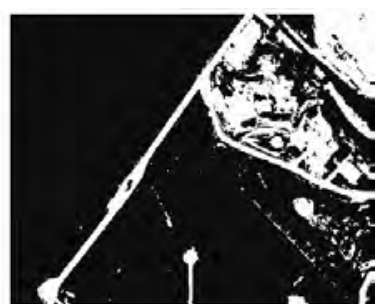

(d)

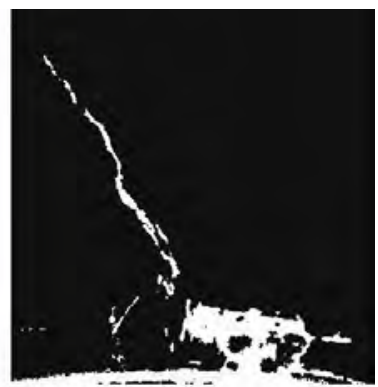

(d)

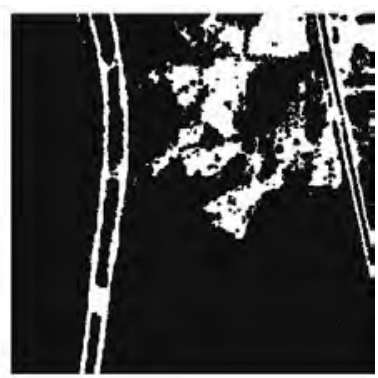

(d)

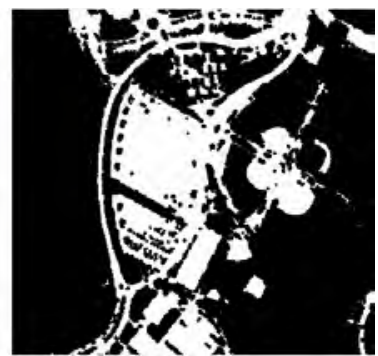

(d)

Figure 1. (a) Initial satellite images, (b) Results of ISODATA clustering, (c) Results of morphological operations. Three clusters are generated, represented by white, black, and gray colors, respectively. However, the identities of the clusters are unknown, (d) Results of classification. Two classes are generated for non-vegetative and vegetative regions, represented by white and black masks, respectively. 


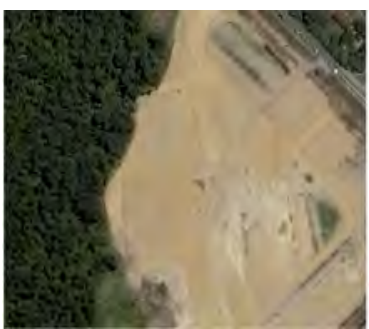

(a)

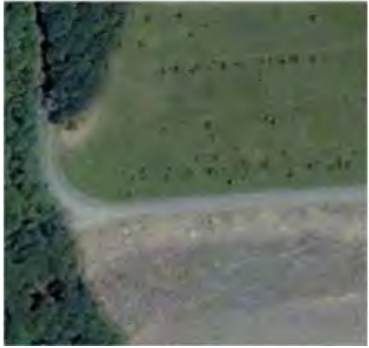

(a)

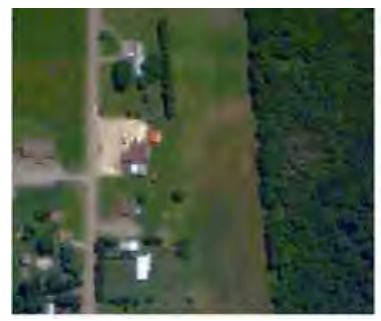

(a)

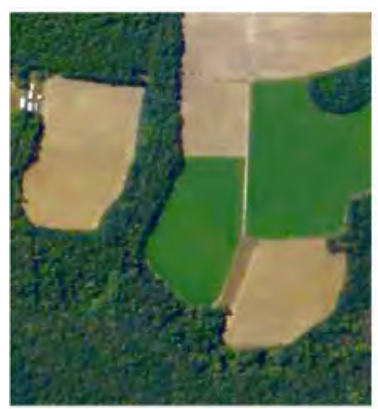

(a)

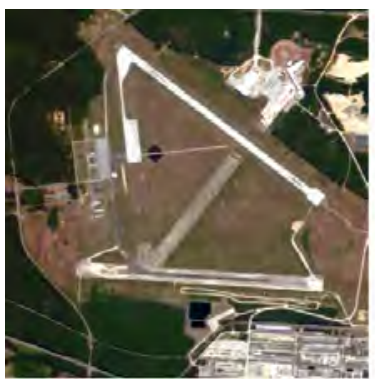

(a)

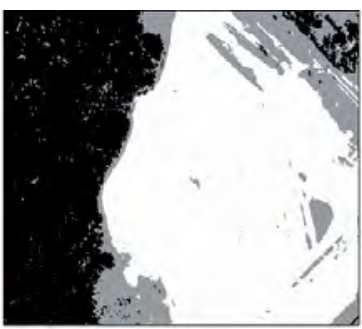

(b)

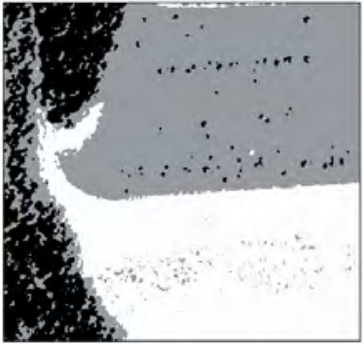

(b)

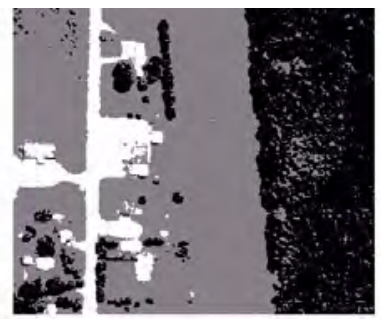

(b)

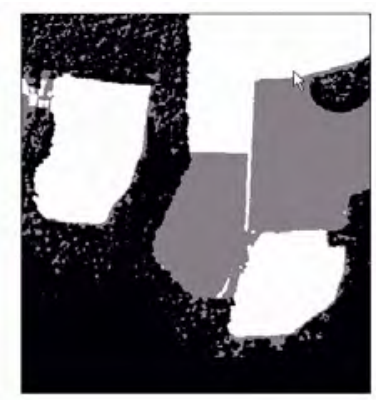

(b)

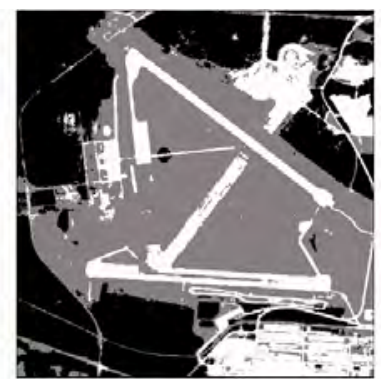

(b)

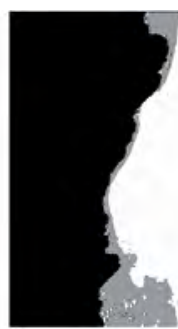

(c)

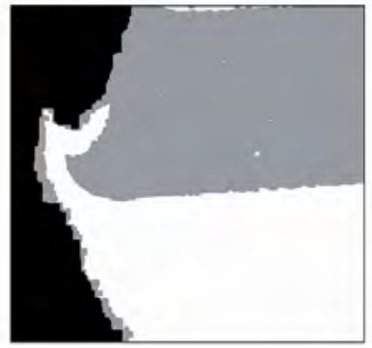

(c)

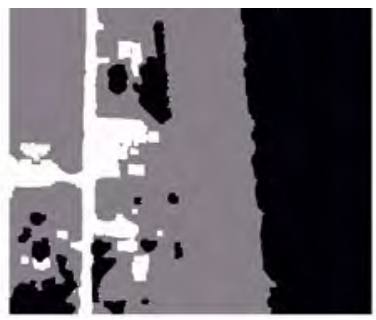

(c)

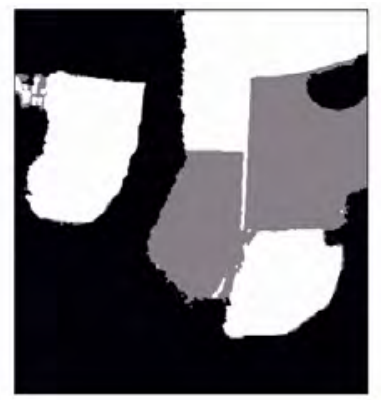

(c)

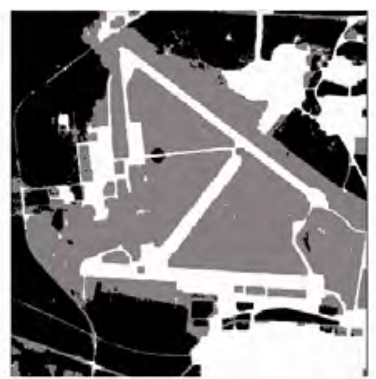

(c)

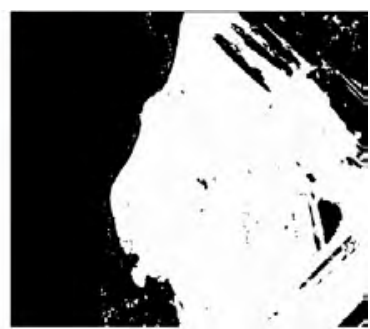

(d)

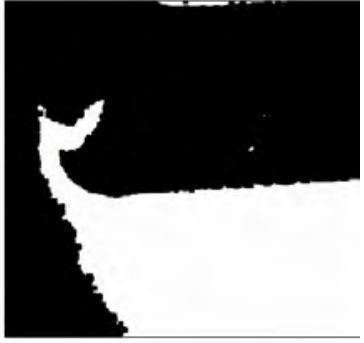

(d)

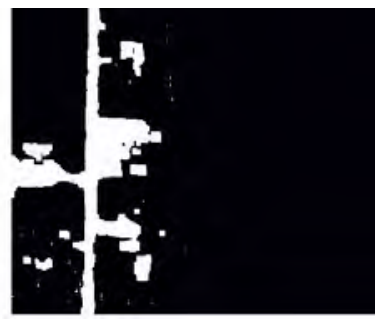

(d)

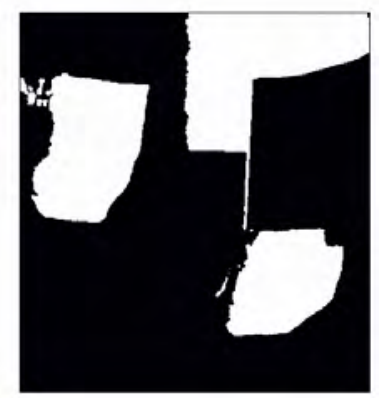

(d)

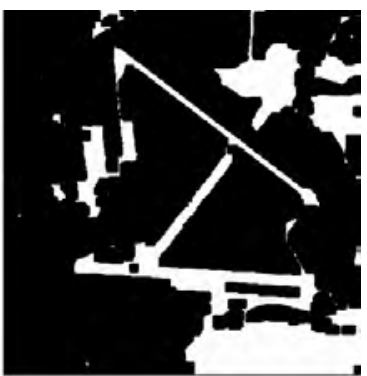

(d)

Figure 2. (a) Initial satellite images. (b) Results of ISODATA clustering. (c) Results of morphological operations. Three clusters are generated, represented by white, black, and gray colors, respectively. However, the identities of the clusters are unknown. (d) Results of classification. Two classes are generated for non-vegetative and vegetative regions, represented by white and black masks, respectively. 


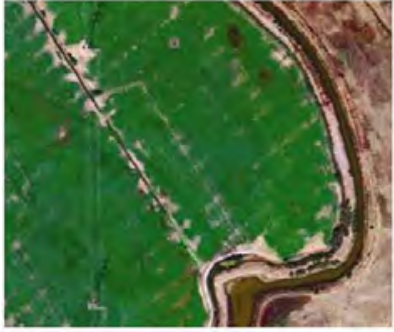

(a)

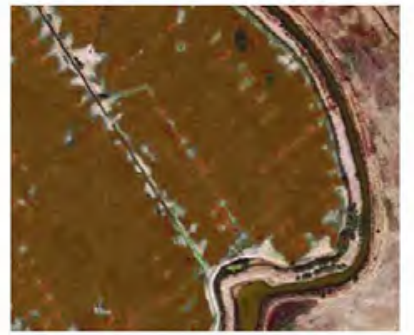

(b)

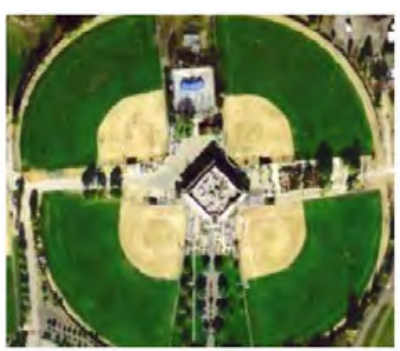

(c)

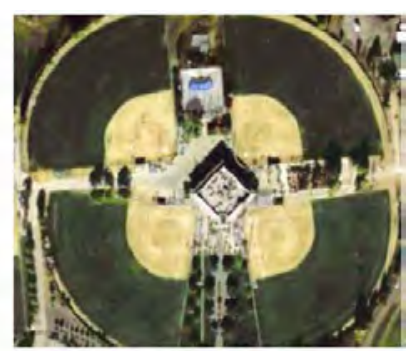

(d)

Figure 5. Brown down effect simulation. (a) and (c) are input target images, while (b) and (d) are output images generated by the proposed method

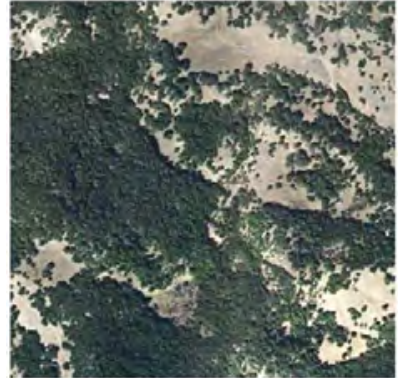

(a)

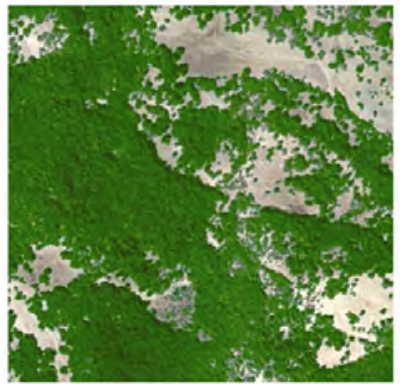

(e)

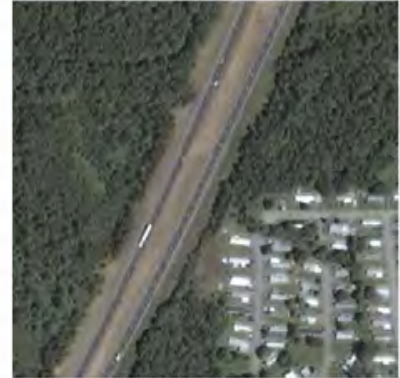

(b)

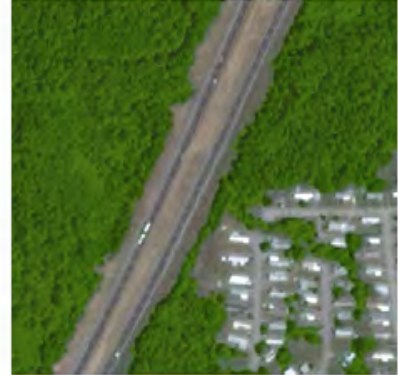

(f)

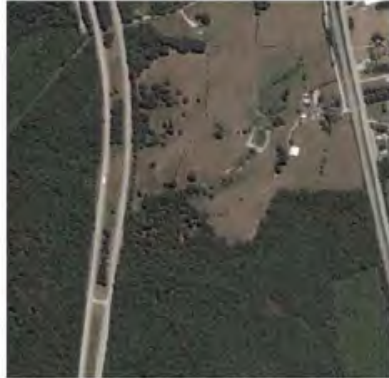

(c)

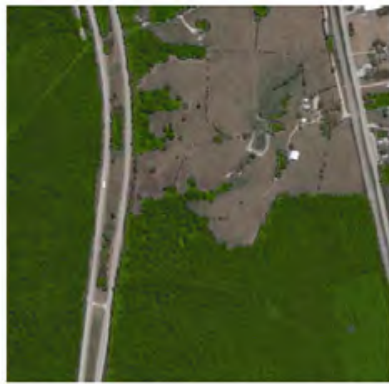

(g)

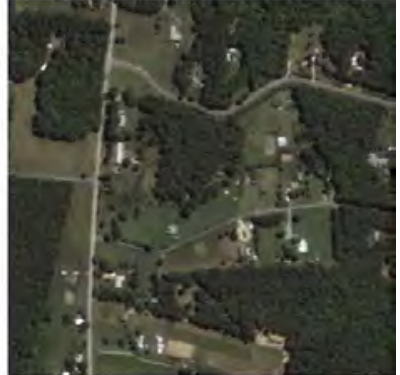

(d)

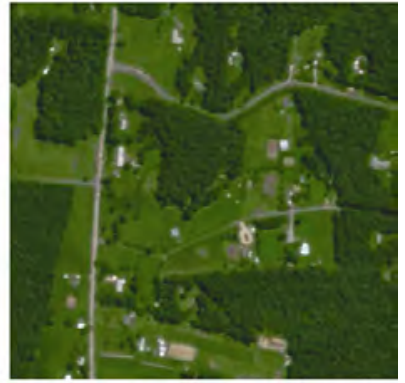

(h)

Figure 6. Green up effect simulation. (a), (c), (e), and (g) are input target images, while (b), (d), (f), and (h) are output images generated by the proposed algorithm.

accuracy. Based on the results, we can reasonably draw the conclusion that it is possible to automatically identify vegetation from other land covers. The MLP classifier can discriminate the two types with an accuracy of up to $96 \%$ in the cross-validation.

Vegetation changes colors due to a number of chemical, physical, and biological processes that occur in different seasons. It is especially worth to note two phenomena, namely, green up and brown down [1]. In spring, leaves emerge and flourish and vegetative growth is rapid, resulting in a greenish appearance, known as green up. From mid-summer to late summer, plant tissues mature, dry, and are harvested, resulting in a brownish appearance, referred to as brown down. There are some other seasonal color changes as well. The im- ages in Figure 5 simulate the brown down effects. Images in Figures 5(a) and (c) are input images, while images in Figures 5(b) and (d) are output images produced by the proposed method. The images in Figure 6 simulate the green up effects. Figures 6(a), (b), (c), and (d) show the input images, while Figures 6(e), (f), (g), and (h) are the output images generated by the proposed method. These results illustrated the effectiveness of the proposed method. These results illustrated the effectiveness of the proposed method. In our current implementation, the vegetation map only contains vegetative areas (and therefore non-vegetative areas). To obtain even better results, the vegetation map needs to be refined, that is, it should indicate different types of vegetation, such as trees and grass. Currently the vegetation maps are gener- 
ated from satellite images in the visible wavelength band. In order to obtain better vegetation maps or feature maps, images from other sources should also be used, such as infrared images in various wavelengths.

\section{Conclusions}

This paper proposed a novel method that alters vegetation colors in satellite images to simulate seasonal changes. The proposed method first generates a vegetation map for pixels corresponding to vegetative areas in satellite images, using ISODATA clustering and vegetation classification. It then generates seasonal color adaptations of a target input image using an image analogies technique. The vegetation map ensures that only the colors of vegetative areas are altered. The proposed method can be used in high performance flight simulations and other applications.

\section{REFERENCES}

[1] J. B. Campbell, "Introduction to remote sensing," The Guilford Press, 4th Edition, 2007.

[2] J. P. Wayman, R. H. Wynne, J. A. Scrivani, and G. A. Burns, "Landsat TM-based forest area estimation using iterative guided spectral class rejection,” Photogrammetric Engineering and Remote Sensing, Vol. 67, pp. 11551166, 2001.

[3] H. Almuallin and T. G. Dietterich, "Learning with many irrelevant features," in Proceedings of AAAI-91, (Anhaim, CA), pp. 547-552, August 1991.

[4] C. Bishop, "Neural networks for pattern recognition," New York: Oxford University Press, 1995.

[5] I. V. Tetko, A. E. P. Villa, and D. J. Livingstone, "Neural network studies. 2: Variable selection,” Journal of Chemistry Information and Computer Science, Vol. 36, No. 4, pp. 794-803, 1996.

[6] K. Kira and L. A. Rendell, "The feature selection problem: traditional methods and a new algorithm," in Proceedings of AAAI-92, (San Jose, CA), pp. 122-126, 1992.
[7] R. Kohavi and G. John, "Wrappers for feature subset selection,” Artificial Intelligence, Vol. 97, No. 1-2, pp. 273-324, 1997.

[8] J. R. Quinlan, "C4.5: Programs for machine learning," San Mateo, California, Morgan Kaufmann, 1993.

[9] L. Breiman, J. Friedman, R. Olshen, and C. Stone, "CART: Classification and regression trees," CBelmont, California, Wadsworth, 1983.

[10] J. Li, M. T. Manry, P. L. Narasimha, and C. Yu, "Feature selection using a piecewise linear network,” IEEE Transactions on Neural Network, Vol. 17, No. 5, pp. 11011105, 2006.

[11] J. Li, M. T. Manry, L. M. Liu, C. Yu, and J. Wei, "Iterative improvement of neural classifiers," Proceedings of the Seventeenth International Conference of the Florida AI Research Society, May 2004.

[12] R. G. Gore, J. Li, M. T. Manry, L. M. Liu, and C. Yu, "Iterative design of neural network classifiers through regression," Special Issue of International Journal on Artificial Intelligence Tools, Vol. 14, No. 1-2, pp. 281-302, 2005.

[13] A. Hertzmann, C. E. Jacobs, N. Oliver, B. Curless, and D. H. Salesin, "Image analogies," in the Proceedings of SIGGRAPH Conference, pp. 327-340, 2001.

[14] S. Chen and R. M. Haralick, "Recursive erosion, dilation, opening, and closing transforms,” IEEE Transactions on Image Processing, Vol. 4, No. 3, 1995.

[15] S. Arya, D. M. Mount, N. S. Netanyahu, R. Silverman, and A. Y. Wu, "An optimal algorithm for approximate nearest neighbor searching in fixed dimensions,” Journal of the ACM, pp. 891-923, 1998.

[16] L. Y. Wei and M. Levoy, "Fast texture synthesis using tree-structured vector quantization," Proceedings of SIGGRAPH Conference, pp. 479-488, July 2000.

[17] M. Ashikhmin, "Synthesizing natural textures," in ACM Symposium on Interactive 3D Graphics, pp. 217-226, March 2001.

[18] A. Hertzmann and C. Jacobs, 2001. http://mrl.nyu.edu/ projects/imageanalogies/lf/. 